Annals of Combinatorics 1 (1997) 353-366

Annals of

Combinalorics

Springer-Verlag 1997

\title{
Kinematic Formulas for Finite Lattices
}

Daniel A. Klain*

School of Mathematics, Georgia Institute of Technology, Atlanta, GA 30332-0160, USA

klain@math.gatech.edu

Received November 10, 1997

AMS Subject Classification: 05A05; 05A10, 05A19, 05A25, 52A22

\begin{abstract}
In analogy to valuation characterizations and kinematic formulas of convex geometry, we develop a combinatorial theory of invariant valuations and kinematic formulas for finite lattices. Combinatorial kinematic formulas are shown to have application to some probabilistic questions, leading in turn to polynomial identities for Möbius functions and Whitney numbers.
\end{abstract}

Keywords: kinematic formula, invariant, valuation, lattice

\section{Introduction}

The lattice structure of the collection of polyconvex sets, that is, the collection of all finite unions of compact convex sets in $\mathbb{R}^{n}$ (also known as the convex ring), provides a crucial link between convex geometry and combinatorial theory. This connection was highlighted by Rota in [20], where a valuation characterization theorem and kinematic formula were derived for the Boolean algebra of subsets of a finite set (see also [11]). In [10], the author extended these notions to the lattice of subspaces of a vector space over a finite field. In this paper we develop a general theory in the broader context of finite posets and lattices of order ideals.

We begin by recalling briefly two important results from classical convex geometry. Denote by $\mathcal{K}^{n}$ the set of all compact convex sets in $\mathbb{R}^{n}$. The set $\mathcal{K}^{n}$ is endowed with the topology induced by the Hausdorff metric on compact sets in $\mathbb{R}^{n}$ (see [22]). A function $\varphi: \mathcal{K}^{n} \longrightarrow \mathbb{R}$ is called a valuation on $\mathcal{K}^{n}$ if $\varphi(\emptyset)=0$, where $\emptyset$ is the empty set, and

$$
\varphi(K \cup L)=\varphi(K)+\varphi(L)-\varphi(K \cap L),
$$

for all $K, L \in \mathcal{K}^{n}$ such that $K \cup L \in \mathcal{K}^{n}$ as well. A valuation $\varphi$ on $\mathcal{K}^{n}$ is said to be rigid motion invariant if $\varphi(g K)=\varphi(K)$ for all rigid motions (translations, rotations, and reflections) $g$ of $\mathbb{R}^{n}$.

* Research supported in part by NSF grant \#DMS 9626688. 
Of particular interest are McMullen's intrinsic volumes [11, 13-15, 22, p. 210], which give invariant extensions of $i$-dimensional volume (on $i$-planes) to polyconvex subsets of $\mathbb{R}^{n}$, where $n \geq i$. Denote by $G(n, i)$ the set of all $i$-dimensional subspaces of $\mathbb{R}^{n}$, equipped with the invariant (Haar) measure $v_{i}$ normalized so that

$$
v_{i}(G(n, i))=\left(\begin{array}{c}
n \\
i
\end{array}\right) \frac{\omega_{n}}{\omega_{i} \omega_{n-i}},
$$

where $\omega_{i}$ is the $i$-dimensional volume of the unit ball in $\mathbb{R}^{i}$. Denote by $V_{i}$ the $i$ dimensional volume in $\mathbb{R}^{i}$. The $i$-volume $V_{i}$ is extended to $i$-th intrinsic volume (also denoted by $V_{i}$ ) on all of $\mathcal{K}^{n}$ by

$$
V_{i}(K)=\int_{G(n, i)} V_{i}(K \mid \xi) d v_{i},
$$

where $K \mid \xi$ denotes the orthogonal projection of $K$ onto the subspace $\xi$. The valuation $V_{i}$ then extends uniquely to the lattice of polyconvex sets via inclusion-exclusion (see, for example, [11,22]).

The valuation $V_{0}$, which takes the value 1 on all non-empty compact convex sets, extends to the Euler characteristic on the lattice of polyconvex sets (see, for example, $[11,14,15,22])$.

Hadwiger's volume theorem states that $V_{n}$ is the only continuous rigid motion invariant valuation on $\mathcal{K}^{n}$ that vanishes on compact convex sets of dimension less than $n$, i.e., on sets with an empty interior. This theorem is easily shown to be equivalent to the following $[7,9,11,22]$.

Theorem 1.1. (Hadwiger's Characterization Theorem) Suppose $\varphi$ is a continuous rigid motion invariant valuation on $\mathcal{K}^{n}$. Then there exist $c_{0}, c_{1}, \ldots, c_{n} \in \mathbb{R}$ such that

$$
\varphi(K)=\sum_{i=0}^{n} c_{i} V_{i}(K)
$$

for all $K \in \mathcal{K}^{n}$.

Hadwiger's characterization leads to simple and straightforward proofs of many classical theorems of integral geometry, an important example of which is the principal kinematic formula.

Denote by $E_{n}$ the group of rigid motions of $\mathbb{R}^{n}$, that is, the indirect sum of the translations group of $\mathbb{R}^{n}$ with the orthogonal group $O(n)$.

Theorem 1.2. (Principal Kinematic Formula) For all polyconvex sets $A$ and $K$,

$$
\int_{E_{n}} V_{0}(A \cap g K) d g=\sum_{i=0}^{n}\left(\begin{array}{c}
n \\
i
\end{array}\right)^{-1} \frac{\omega_{i} \omega_{n-i}}{\omega_{n}} V_{i}(A) V_{n-i}(K) .
$$

The integral in (1.2) is taken with respect to the indirect sum of the Lebesgue measure on $\mathbb{R}^{n}$ with the Haar probability measure on $O(n)$. For compact convex sets $A$ and $K$, this integral has an evident geometric interpretation as the measure of the set of all 
$g \in E_{n}$ such that $A \cap g K \neq \emptyset$. Alternatively, one may think of (1.2) as the "measure" of all convex sets $g K$ in $\mathbb{R}^{n}$ congruent to $K$ that meet $A$.

Theorem 1.2 is one of a family of kinematic formulas for valuations on polyconvex sets, attributed in origin to Blaschke [1], Chern [3], and Santaló [21]. The techniques of the present work are inspired by those of Hadwiger [7] and Rota [20] (see also [11]). Kinematic formulas remain a topic of current interest in convex and integral geometry (see $[4,5,8,11,22,23,26,27])$.

\section{Invariant Valuations on Finite Lattices}

Let $P$ denote a finite set, partially ordered by the relation $\leq$, and with a minimal element 0 . We shall also refer to the partially ordered set $P$ as a poset. An automorphism of a poset $P$ is a bijective function $g: P \longrightarrow P$ which preserves the ordering $\leq$. The collection of all automorphisms of a poset $P$ forms a group under composition of functions, denoted by $\operatorname{Aut}(P)$. If $G$ is a subgroup of $\operatorname{Aut}(P)$, then $G$ acts on $P$ by evaluation of the functions $g \in G$ at elements $x \in P$.

For $x \in P$, denote

$$
U(x)=\{g x: g \in G\},
$$

that is, the orbit of $x$ under the action of $G$. Let $\mathcal{U}(G ; P)$ denote the collection of all orbits in $P$ of the action of the group $G$, except the singleton orbit $\{0\}$ of the minimal element 0 . We shall refer to $\mathcal{U}(G ; P)$ as $\mathcal{U}$ where no confusion is possible. Denote

$$
\operatorname{Stab}(x)=\{g \in G: g x=x\},
$$

that is, the stabilizer of $x$ under the action of $G$. Recall that $\operatorname{Stab}(x)$ is a subgroup of $G$.

If $A$ is a finite set, denote by $|A|$ the number of elements of $A$. The orbit and stabilizer of an element $x \in P$ satisfy the identity:

$$
|U(x)||\operatorname{Stab}(x)|=|G| .
$$

An order ideal $I$ is a subset of $P$ such that, if $x \in A$ and $y \leq x$, then $y \in A$. An order ideal is a partially ordered set in the order induced by $P$. An order ideal having exactly one maximal element is called a principal ideal.

The (set-theoretic) union and intersection of any number of order ideals is again an order ideal. Thus, the set $J(P)$ of all order ideals in $P$ is a distributive lattice and we can study valuations on $J(P)$.

A function $\varphi: J(P) \longrightarrow \mathbb{R}$ is called a valuation if $\varphi(\{0\})=0$ and

$$
\varphi(A \cup B)+\varphi(A \cap B)=\varphi(A)+\varphi(B),
$$

for all $A, B \in J(P)$.

For $x \in P$, denote by $\bar{x}$ the principal ideal whose maximal element is $x$, that is, the set of all $y \in P$ such that $y \leq x$.

It is well known (or see $[11,19]$ ) that every valuation $\varphi$ on $J(P)$ extends uniquely to a valuation, again denoted by $\varphi$, on the Boolean algebra $B(P)$ of all subsets of $P$, which is generated by $J(P)$. Such a valuation is evidently determined by its value on the one element subsets of $B(P)$, that is, by arbitrarily assigning a value $\varphi(\{x\})$ for each $x \in L$. 
Let $x \in P$ and let $I_{1}, I_{2}, \ldots, I_{m}$ be the maximal principal ideals $I_{k} \subseteq \bar{x}$ such that $I_{k} \neq \bar{x}$. Then

$$
\varphi(\{x\})=\varphi(\bar{x})-\varphi\left(I_{1} \cup I_{2} \cup \cdots \cup I_{m}\right) .
$$

The right-hand side can be computed in terms of principal ideals of lower order by the inclusion-exclusion principle (1.1). Thus, by induction on the partial ordering, we have the following theorem (due to Rota $[12,19]$ ).

Theorem 2.1. Every valuation $\varphi$ on the distributive lattice $J(P)$ of all order ideals is uniquely determined by the values $\varphi(\bar{x})$, over all $x \in P$. The values $\varphi(\bar{x})$ may be arbitrarily assigned.

Suppose $G$ is a group acting on the poset $P$. For $I \in J(P)$, the set

$$
g I=\{g x: x \in I\}
$$

is also an order ideal of $P$. It is easily verified that (2.1) defines a group action of $G$ on the lattice $J(P)$. We say that this $G$-action on $J(P)$ is induced by the action of $G$ on $P$.

A valuation $\varphi$ on $J(P)$ is called invariant if it is invariant under the action of group $G$, that is, if $\varphi(I)=\varphi(g I)$ for every order ideal $I \in J(P)$ and all $g \in G$.

Among the most important invariant valuations on any lattice is the Euler characteristic, which we now define. The following is an immediate consequence of Theorem 2.1. (See also [19].)

Theorem 2.2. There exists a unique invariant valuation $\varphi$ on $J(P)$ called the Euler characteristic, such that $\chi(\bar{x})=1$ for every principal ideal $\bar{x}$ with $x>0$ and $\chi(\overline{\{0\}})=0$.

Recall that the Euler characteristic of the distributive lattice $J(P)$ of order ideals of a poset $P$ satisfies

$$
\chi(I)=-\sum_{x \in I, x>0} \mu(0, x)
$$

where 0 denotes the minimal element of $P$ and $\mu$ is the Möbius function of $P$ (see [12, $18,19,24$, p.120, 25]).

For $U \in \mathcal{U}(P ; G)$, define

$$
\varphi_{\mathrm{U}}(\bar{x})=|\bar{x} \cap U|
$$

and extend $\varphi_{\mathrm{U}}$ to all of $J(P)$ by Theorem 2.1. For every order ideal $I$,

$$
\varphi_{\mathrm{U}}(I)=|I \cap U| .
$$

In other words, the valuation $\varphi_{U}$ counts the number of elements of the orbit $U$ contained in an order ideal.

Note that if $y \in U(x)$, then $\mu(0, x)=\mu(0, y)$. This follows from the fact that $y=g x$ for some automorphism $g$ of $P$. Therefore, we denote by $\mu(U)$ the value of $\mu(0, x)$ for any $x \in U$. It now follows from (2.2) that

$$
\chi(I)=-\sum_{U \in \mathcal{U}(P ; G)} \sum_{x \in U} \mu(0, x)=-\sum_{U \in \mathcal{U}(P ; G)} \mu(U) \varphi_{\mathrm{U}}(I),
$$


for any order ideal $I$.

More generally, we have the following combinatorial analogue of Hadwiger's characterization theorem for invariant valuations.

Theorem 2.3. (Basis Theorem) The invariant valuations $\left\{\varphi_{U}: U \in \mathcal{U}(P ; G)\right\}$ span the vector space of all valuations $\varphi$ on $J(P)$ that are invariant under the action of $G$.

Proof. Suppose $\varphi$ is an invariant valuation on $J(P)$. Extend $\varphi$ to all of $B(P)$. The extended valuation, which is still denoted by $\varphi$, is again invariant. If $U(x)=U(y)=$ $U$, then there exists an automorphism $g$ of $P$ such that $g x=y$. Therefore, $\varphi(\{x\})=$ $\varphi(\{y\})=c_{\mathrm{U}}$, for some constant $c_{\mathrm{U}} \in \mathbb{R}$. Thus, the valuation

$$
\varphi-\sum_{U \in \mathcal{U}(P ; G)} c_{U} \varphi_{U}
$$

vanishes on all singleton sets $\{x\}$ for all $x \in P$, and consequently vanishes on all of $B(P)$.

In order to compute the coefficients $c_{\mathrm{U}}$ given by the Basis Theorem 2.3, note that if $x \in U$, then

$$
\varphi(\{x\})=\sum_{U \in \mathcal{U}(P ; G)} c_{\mathrm{U}} \varphi_{\mathrm{U}}(\{x\})=c_{\mathrm{U}} .
$$

If we know the values of $\varphi(\{x\})$ for some $x$ in each orbit $U$, then we are done.

However, a valuation $\varphi$ is often given in terms of its values on principal ideals $\bar{x}$ for $x \in P$ (as in, for example, Theorem 2.1). In order to compute the values $\varphi(\{x\})$, given the values $\varphi(\bar{x})$, we use Möbius inversion. Recall that the extension of a valuation $\varphi$ on $J(P)$ to all of $B(P)$ is given inductively by

$$
\varphi(\{0\})=0
$$

and

$$
\varphi(\{x\})=\varphi(\bar{x})-\sum_{y<x} \varphi(\{y\}),
$$

so that

$$
\varphi(\bar{x})=\sum_{y \leq x} \varphi(\{y\})
$$

for all $x \in P$. Applying Möbius inversion to (2.5) yields

$$
\varphi(\{x\})=\sum_{y \leq x} \mu(y, x) \varphi(\bar{y}) .
$$

Combining (2.6) with (2.4) along with the invariance of $\varphi$, we obtain

$$
c_{\mathrm{U}}=\varphi\left(\left\{x_{\mathrm{U}}\right\}\right)=\sum_{y \leq x_{\mathrm{U}}} \mu\left(y, x_{\mathrm{U}}\right) \varphi(\bar{y}),
$$

for each $U \in \mathcal{U}$. 


\section{Kinematic Formulas for the Lattice of Order Ideals}

As an application of the Basis Theorem 2.3 we shall derive a combinatorial analogue of the principal kinematic formula (1.2).

One way to construct invariant valuations on $J(P)$ is the following. Start with any valuation $\varphi$ on $J(P)$ such that $\varphi(\{\emptyset\})=0$ and let $A$ be any order ideal. For any order ideal $B$, define

$$
\varphi(A ; B)=\frac{1}{|G|} \sum_{g} \varphi(A \cap g B),
$$

where $g$ varies over the group $G$. For fixed $A$, the set function $\varphi(A ; B)$ is a valuation in the variable $B$; in fact, it is an invariant valuation, since

$$
\begin{aligned}
\varphi\left(A ; g_{0} B\right) & =\frac{1}{|G|} \sum_{g} \varphi\left(A \cap g g_{0} B\right) \\
& =\frac{1}{|G|} \sum_{g} \varphi(A \cap g B),
\end{aligned}
$$

for each automorphism $g_{0} \in G$. By Theorem 2.3, the functional $\varphi(A ; B)$ can be expressed as a linear combination of the valuations $\varphi_{\mathrm{U}}$, with coefficients $\alpha_{\mathrm{U}}(A)$ depending on $A$ :

$$
\varphi(A ; B)=\sum_{U \in \mathcal{U}} \alpha_{\mathrm{U}}(A) \varphi_{\mathrm{U}}(B) .
$$

Meanwhile, for fixed $B$, the set function $\varphi(A ; B)$ is a valuation in the variable $A$. From this it follows that each of the coefficients $\alpha_{U}(A)$ is a valuation in the variable $A$.

Now consider the case when $\varphi$ is an invariant valuation. If so, then

$$
\begin{aligned}
\varphi(A ; B) & =\frac{1}{|G|} \sum_{g} \varphi(A \cap g B)=\frac{1}{|G|} \sum_{g} \varphi\left(g^{-1} A \cap B\right) \\
& =\frac{1}{|G|} \sum_{g} \varphi(g A \cap B)=\varphi(B ; A) .
\end{aligned}
$$

It follows that $\alpha_{\mathrm{U}}(A)$ is an invariant valuation in the parameter $A$, so that

$$
\varphi(A ; B)=\sum_{U, V \in \mathcal{U}} \alpha_{\mathrm{UV}} \varphi_{\mathrm{U}}(A) \varphi_{\mathrm{v}}(B)
$$

Since $\varphi(A ; B)=\varphi(B ; A)$, it is evident that $\alpha_{\mathrm{Uv}}=\alpha_{\mathrm{vU}}$. It turns out that most of the constants $\alpha_{\mathrm{Uv}}$ are equal to zero. To compute the coefficients $\alpha_{\mathrm{Uv}}$, extend the valuation $\varphi$ to the Boolean algebra $B(P)$ generated by $J(P)$ and let $c_{\mathrm{U}}$ denote the value of $\varphi$ on a singleton set in $B(P)$ whose single element lies in the orbit $U$ (that is, $c_{\mathrm{U}}=\varphi(\{x\})$, for any $x \in U$ ). Recall that the values $c_{\mathrm{U}}$ may be obtained from (2.4) or (2.7).

Theorem 3.1. (General Kinematic Formula) Suppose $\varphi$ is an invariant valuation on $J(P)$. For all $A, B \in J(P)$,

$$
\frac{1}{|G|} \sum_{g} \varphi(A \cap g B)=\sum_{U \in \mathcal{U}} \frac{1}{|U|} c_{U} \varphi_{U}(A) \varphi_{U}(B) .
$$


When $P$ is a modular lattice and the orbits $U$ coincide with the grades of $P$, the values $|U|$ are sometimes called the Whitney numbers (of the second kind) for $P$.

Proof. Suppose $x_{\mathrm{v}}, y_{\mathrm{v}} \in P$ lie in orbits $U$ and $V$ respectively. Let $A=\left\{x_{\mathrm{U}}\right\}$ and $B=\left\{y_{\mathrm{v}}\right\}$. For any $g \in G$, the set $A \cap g B=\emptyset$ if $U \neq V$. If $U=V$, then $A \cap g B=\emptyset$ if $x_{\mathrm{U}} \neq g y_{\mathrm{v}}$, while there are $\left|\operatorname{Stab}\left(x_{\mathrm{U}}\right)\right|$ automorphisms $g$ of $V$ such that $x_{\mathrm{U}}=g y_{\mathrm{v}}$. Hence, we have

$$
\varphi(A ; B)=\frac{1}{|G|} \sum_{g} \varphi(A \cap g B)=\frac{\left|\operatorname{Stab}\left(x_{\mathrm{U}}\right)\right|}{|G|} \varphi(A)=\frac{1}{|U|} c_{\mathrm{U}} .
$$

Meanwhile, $\varphi_{\mathrm{U}_{0}}(A)=1$ if $U_{0}=U$; otherwise, it is equal to zero. Similarly, $\varphi_{\mathrm{v}_{0}}(B)=1$ if $V_{0}=V$; otherwise, it is equal to zero. Hence,

$$
\varphi(A ; B)=\sum_{U_{0}, V_{0} \in \mathcal{U}} \alpha_{\mathrm{U}_{0} \mathrm{v}_{0}} \varphi_{\mathrm{U}_{0}}(A) \varphi_{\mathrm{v}_{0}}(B)=\alpha_{\mathrm{UV}} .
$$

Therefore, $\alpha_{\mathrm{UV}}=(1 /|U|) c_{\mathrm{U}}$ if $U=V$, otherwise, it is equal to zero.

For example, if $\varphi=\varphi_{\mathrm{U}}$, then (3.2) becomes

$$
\frac{1}{|G|} \sum_{g} \varphi_{\mathrm{U}}(A \cap g B)=\frac{1}{|U|} \varphi_{\mathrm{U}}(A) \varphi_{\mathrm{U}}(B) .
$$

The basis theorem implies that every kinematic formula for an invariant valuation can be expressed by taking linear combinations of the identity (3.3).

The case $\varphi=\chi$ is of particular interest. The Euler formula (2.3) implies that $\chi\left(\left\{x_{\mathrm{U}}\right\}\right)=-\mu(U)$. Theorem 3.1 then specializes to the following combinatorial analogue of Theorem 1.2.

Theorem 3.2. (Principal Kinematic Formula) For all $A, B \in J(P)$,

$$
\frac{1}{|G|} \sum_{g} \chi(A \cap g B)=\sum_{U \in \mathcal{U}}-\mu(U) \frac{1}{|U|} \varphi_{U}(A) \varphi_{U}(B) .
$$

The probability that a randomly chosen principal ideal $\bar{y}$ shall meet a fixed principal ideal $\bar{x}$ is now given by

$$
\frac{1}{|G|} \sum_{g} \chi(\bar{x} \cap g \bar{y})=\sum_{U \in \mathcal{U}}-\mu(U) \frac{1}{|U|} \varphi_{\mathrm{U}}(\bar{x}) \varphi_{\mathrm{U}}(\bar{y}) .
$$

By combining (3.3) and (3.5) with standard probabilistic arguments, one may derive new combinatorial identities, as is demonstrated in the examples that follow.

\section{Examples and Applications}

We now apply the the theory of invariant valuations to central examples of posets in enumerative combinatorics. 


\subsection{Finite Boolean Algebras}

Consider the lattice $B_{n}$ of all subsets of $\{1,2, \ldots, n\}$, a Boolean algebra. The collection $B_{n}$ is a poset when ordered by inclusion of subsets. Let $G=S_{n}$, the group of permutations on $n$ elements. The orbits of the action of $G$ are the collections $B_{n}^{k}$ of $k$-element subsets of $\{1,2, \ldots, n\}$, having size $\left|B_{n}^{k}\right|=\left(\begin{array}{l}n \\ k\end{array}\right)$.

The Möbius function of the poset $B_{n}$ is given by

$$
\mu(x, y)=(-1)^{|y|-|x|}
$$

for $x, y \in B_{n}$ such that $x \subseteq y$. The Basis Theorem 2.3 implies that all invariant valuations on $J\left(B_{n}\right)$ are linear combinations of the valuations $\varphi_{i}$, given by

$$
\varphi_{i}(I)=\left|I \cap B_{n}^{i}\right|,
$$

for all $I \in J\left(B_{n}\right)$ and $i=1, \ldots, n$.

It then follows from Theorem 3.2 that

$$
\frac{1}{n !} \sum_{g \in S_{n}} \chi(A \cap g B)=\sum_{i=1}^{n}(-1)^{i+1}\left(\begin{array}{c}
n \\
i
\end{array}\right)^{-1} \varphi_{i}(A) \varphi_{i}(B),
$$

for all $A, B \in J\left(B_{n}\right)$. If $A=\bar{x}_{k}$ and $B=\bar{y}_{l}$, then the left-hand side of (4.1) expresses the probability that a randomly chosen $l$-simplex will meet a given $k$-simplex. Using elementary probabilistic reasoning, one can easily check that the probability that a random $l$-simplex does not meet a given fixed $k$-simplex is

$$
\left(\begin{array}{l}
n \\
l
\end{array}\right)^{-1}\left(\begin{array}{c}
n-k \\
l
\end{array}\right)
$$

From (4.1), we then obtain

$$
1-\left(\begin{array}{l}
n \\
l
\end{array}\right)^{-1}\left(\begin{array}{c}
n-k \\
l
\end{array}\right)=\frac{1}{n !} \sum_{g \in S_{n}} \chi\left(\bar{x}_{k} \cap g \bar{y}_{l}\right)=\sum_{i=1}^{n}(-1)^{i+1}\left(\begin{array}{l}
n \\
i
\end{array}\right)^{-1}\left(\begin{array}{l}
k \\
i
\end{array}\right)\left(\begin{array}{l}
l \\
i
\end{array}\right) .
$$

By adding the term corresponding $i=0$ to both sides of (4.2) and multiplying by -1 , we obtain the identity

$$
\sum_{i=0}^{n}(-1)^{i}\left(\begin{array}{l}
n \\
i
\end{array}\right)^{-1}\left(\begin{array}{l}
l \\
i
\end{array}\right)\left(\begin{array}{l}
k \\
i
\end{array}\right)=\left(\begin{array}{l}
n \\
l
\end{array}\right)^{-1}\left(\begin{array}{c}
n-k \\
l
\end{array}\right)
$$

for all positive integers $0 \leq k, l \leq n$.

Note that if $k+l>n$, then $\left(\begin{array}{c}n-k \\ l\end{array}\right)=0$. In the preceding argument, this corresponds to the case in which the two sets $g y_{l}$ and $x_{k}$ have non-empty overlap for any permutation $g$, i.e., the case in which $x_{k} \cap g y_{l}=\emptyset$ with probability zero.

The left-hand side of (4.1) can be computed once again using standard combinatorial arguments. Let $x_{k} \in B_{n}$. The number of $l$-sets $y \in B_{n}$ such that $x_{k} \cap y$ is a set of size $i$ is given by

$$
\left(\begin{array}{c}
k \\
i
\end{array}\right)\left(\begin{array}{c}
n-k \\
l-i
\end{array}\right)
$$


On combining this with (4.1), we obtain the identity

$$
\sum_{i=1}^{n}(-1)^{i+1}\left(\begin{array}{l}
n \\
i
\end{array}\right)^{-1}\left(\begin{array}{l}
k \\
i
\end{array}\right)\left(\begin{array}{l}
l \\
i
\end{array}\right)=\left(\begin{array}{l}
n \\
l
\end{array}\right)^{-1} \sum_{i=1}^{n}\left(\begin{array}{l}
k \\
i
\end{array}\right)\left(\begin{array}{c}
n-k \\
l-i
\end{array}\right) .
$$

On simplifying, we then obtain

$$
\sum_{i=1}^{n}\left(\begin{array}{l}
k \\
i
\end{array}\right)\left(\left(\begin{array}{c}
n-k \\
l-i
\end{array}\right)+(-1)^{i}\left(\begin{array}{c}
n-i \\
l-i
\end{array}\right)\right)=0
$$

Similarly, we use the identity (3.3) to compute the expected value of $\varphi_{m}\left(x_{k} \cap g y_{l}\right)$, yielding the identity

$$
\sum_{i=1}^{n}\left(\begin{array}{l}
n \\
l
\end{array}\right)^{-1}\left(\begin{array}{l}
k \\
i
\end{array}\right)\left(\begin{array}{c}
n-k \\
l-i
\end{array}\right)\left(\begin{array}{l}
i \\
m
\end{array}\right)=\left(\begin{array}{l}
n \\
m
\end{array}\right)^{-1}\left(\begin{array}{l}
k \\
m
\end{array}\right)\left(\begin{array}{c}
l \\
m
\end{array}\right),
$$

for all $0 \leq k, l, m \leq n$. The left-hand side of (4.5) is obtained from the definition of expectation, while the right-hand side follows immediately from (3.3).

\subsection{Finite Vector Spaces}

Denote by $L_{n}(q)$ the set of all subspaces of a vector space $V$ of dimension $n$ over a finite field of order $q$. The collection $L_{n}(q)$ is a poset when ordered by the inclusion of subspaces. Let $G=G L_{n}(q)$, the group of linear automorphisms of $V$. The orbits of the action of $G$ are the collections $L_{n}^{k}(q)$ of $k$-dimensional subspaces of $V$, having size

$$
\left|L_{n}^{k}(q)\right|=\left(\begin{array}{l}
n \\
k
\end{array}\right)_{q}=\frac{\left(q^{n}-1\right) \cdots\left(q^{n-k+1}-1\right)}{\left(q^{k}-1\right) \cdots(q-1)}
$$

the Gaussian coefficients. Recall also that

$$
\left|G L_{n}(q)\right|=\left(q^{n}-1\right)\left(q^{n}-q\right) \cdots\left(q^{n}-q^{n-1}\right) .
$$

The Möbius function of the poset $L_{n}(q)$ is given by

$$
\mu(x, y)=(-1)^{\operatorname{dim}(y)-\operatorname{dim}(x)} q^{\left(\frac{\operatorname{dim}(y)-\operatorname{dim}(x)}{2}\right),}
$$

for $x, y \in L_{n}(q)$ such that $x \subseteq y$.

By the Basis Theorem 2.3, the collection of valuations $\left\{\varphi_{i}\right\}_{i=1}^{n}$ on $J\left(B_{n}\right)$ given by

$$
\varphi_{i}(I)=\left|I \cap L_{n}^{i}(q)\right|
$$

is a basis for the space of invariant valuations on $L_{n}(q)$. From Theorem 3.2, we then obtain

$$
\frac{1}{\left|G L_{n}(q)\right|} \sum_{g} \chi(A \cap g B)=\sum_{i=1}^{n}(-1)^{i+1} q^{\left(\begin{array}{c}
i \\
2
\end{array}\right)}\left(\begin{array}{l}
n \\
i
\end{array}\right)_{q}^{-1} \varphi_{i}(A) \varphi_{i}(B),
$$

for all $A, B \in J\left(L_{n}(q)\right)$. 
Similarly, the kinematic formula (3.3) now becomes

$$
\frac{1}{\left|G L_{n}(q)\right|} \sum_{g} \varphi_{i}(A \cap g B)=\left(\begin{array}{c}
n \\
i
\end{array}\right)_{q}^{-1} \varphi_{i}(A) \varphi_{i}(B)
$$

for all $A, B \in J\left(L_{n}(q)\right)$ and for each $i=1, \ldots, n$. Let $x_{k}$ and $y_{l}$ be subspaces of $V$ having dimensions $k$ and $l$, respectively. In the case when $A=\bar{x}_{k}$ and $B=\bar{y}_{l}$, the formula (4.8) gives the expected number of $i$-dimensional subspaces inside the intersection of the $k$-dimensional subspace $x_{k}$ with a random $l$-dimensional subspace $g x_{l}$.

By applying elementary probabilistic reasoning to (4.7), we obtain the $q$-analogue of (4.3), to wit:

$$
\sum_{i=0}^{n}(-1)^{i} q^{\left(\begin{array}{c}
i \\
2
\end{array}\right)}\left(\begin{array}{l}
n \\
i
\end{array}\right)_{q}^{-1}\left(\begin{array}{l}
k \\
i
\end{array}\right)_{q}\left(\begin{array}{l}
l \\
i
\end{array}\right)_{q}=q^{k l}\left(\begin{array}{l}
n \\
l
\end{array}\right)_{q}^{-1}\left(\begin{array}{c}
n-k \\
l
\end{array}\right)_{q} .
$$

for all positive integers $n, q$ and all $0 \leq k, l \leq n$. This formula was originally obtained by Chen and Rota (see [2, (4.4)] and also [12, p. 273]).

For additional details, see [10]. See also [6, 12, 24, pp. 126-127, 25, pp. 291-197] for a treatment of $q$-analogues, Gaussian coefficients, and the Möbius function (4.6).

\subsection{The Lattice of Partitions (Order Ideals)}

Denote by $\Pi_{n}$ the set of all partitions of the set $\{1,2, \ldots, n\}$. The collection $\Pi_{n}$ is a poset when ordered by a refinement of partitions. Specifically, we have $\pi \leq \sigma$ in $\Pi_{n}$ provided each block of the partition $\pi$ is contained inside a block of $\sigma$. We denote by $|\pi|$ the number of blocks in the partition $\pi$.

Let $G=S_{n}$, the group of permutations of $\{1,2, \ldots, n\}$. The orbits of $\Pi_{n}$ under the action of $G$ are collections of partitions having the same shape. Let $\pi \in \Pi_{n}$ and let $a_{i}$ denote the number of blocks of $\pi$ of size $i$. Then the orbit $U(\pi)$ has size

$$
|U(\pi)|=\frac{n !}{(1 !)^{a_{1}} a_{1} ! \cdots(n !)^{a_{n}} a_{n} !}=n ! \prod_{k=1}^{n} \frac{1}{(k !)^{a_{k}} a_{k} !} .
$$

For $\pi, \sigma \in \Pi_{n}$ such that $\pi \leq \sigma$, the Möbius function $\mu(\pi, \sigma)$ is given by

$$
\mu(\pi, \sigma)=(-1)^{|\pi|-|\sigma|} \prod_{P \in \sigma}\left(n_{P}-1\right) !,
$$

where $n_{P}$ denotes the number of blocks of $\pi$ contained in the block $P$. For a derivation of (4.9), see [25, p. 301].

Note that if $\pi=0_{\Pi_{n}}$, the discrete partition, then (4.9) becomes

$$
\mu\left(0_{\Pi_{n}}, \sigma\right)=(-1)^{n-|\sigma|} \prod_{P \in \sigma}(|P|-1) !=(-1)^{n-|\sigma|} \prod_{k=1}^{n}(k-1) !^{a_{k}},
$$

where $a_{k}$ denotes the number of blocks of $\sigma$ of size $k$. 
Combining (4.10) with Theorem 3.2 we obtain

$$
\begin{aligned}
& \frac{1}{n !} \sum_{g} \chi(A \cap g B) \\
& =\sum_{a_{1}+2 a_{2}+\cdots+n a_{n}=n}(-1)^{n-|\sigma|+1}\left(\prod_{k=1}^{n}(k-1) !^{a_{k}}\right) \frac{1}{n !}\left(\prod_{k=1}^{n}(k !)^{a_{k}} a_{k} !\right) \varphi_{\mathrm{U}}(A) \varphi_{\mathrm{U}}(B) \\
& =\sum_{a_{1}+2 a_{2}+\cdots+n a_{n}=n} \frac{(-1)^{n-|\sigma|+1}}{n !}\left(\prod_{k=1}^{n}((k-1) !)^{a_{k}}(k !)^{a_{k}} a_{k} !\right) \varphi_{\mathrm{U}}(A) \varphi_{\mathrm{U}}(B)
\end{aligned}
$$

for all $A, B \in J\left(\Pi_{n}\right)$, and where $|\sigma|=a_{1}+\cdots+a_{n}$.

Unfortunately, the values of $\varphi_{U}(A)$ are very difficult to compute for a typical order ideal $A$ in $\Pi_{n}$.

\subsection{The Lattice of Partitions (Filters)}

Unlike the previous examples, the lattice $\Pi_{n}$ is not self-dual. Denote by $\Pi_{n}^{*}$ the lattice dual to $\Pi_{n}$, in which all order relations in $\Pi_{n}$ are reversed. Denote by $1_{\Pi_{n}}$ the indiscrete partition consisting of a single block, $1_{\Pi_{n}}=\{\{1, \ldots, n\}\}$. The Möbius function for $\Pi_{n}^{*}$ is then given by

$$
\mu\left(0_{\Pi_{n}^{*}}, \sigma\right)=\mu\left(\sigma, 1_{\Pi_{n}}\right)=(-1)^{|\sigma|-1} \prod_{B \in 1_{\Pi_{n}}}\left(n_{B}-1\right) !=(-1)^{|\sigma|-1}(|\sigma|-1) ! .
$$

It follows from Theorem 3.2 that

$$
\frac{1}{n !} \sum_{g \in S_{n}} \chi(A \cap g B)=\sum_{a_{1}+2 a_{2}+\cdots+n a_{n}=n}(-1)^{|\sigma|} \frac{(|\sigma|-1) !}{n !}\left(\prod_{k=1}^{n}(k !)^{a_{k}} a_{k} !\right) \varphi_{\mathrm{U}}(A) \varphi_{\mathrm{U}}(B) .
$$

Once again the values of $\varphi_{U}(A)$ are very difficult to compute for a typical order ideal $A$ in $\Pi_{n}^{*}$ (a filter in $\Pi_{n}$ ).

\subsection{The Lattice of Faces of an $n$-cube}

Let $C_{n}$ denote the $n$-dimensional unit cube in $\mathbb{R}^{n}$. Let $C^{n}$ denote the regular cell decomposition of the cube $C_{n}$ into lower-dimensional unit cubes. The cell complex $C^{n}$ is partially ordered by set inclusion of the faces of $C_{n}$. The null set $\emptyset$ is included as the minimal element of the lattice $C^{n}$. For a description of the lattice structure of $C^{n}$, see $[16,17]$. See also [12] for an extensive bibliography.

The Möbius function of the lattice $C^{n}$ is characterized as follows.

Proposition 4.1. Let $C, D \in C^{n}$ be any two cells with $C \subseteq D$ and $C \neq \emptyset$. If $\operatorname{dim} C=k$ and $\operatorname{dim} D=l$, then

$$
\mu(C, D)=(-1)^{l-k} \text { and } \mu(\emptyset, C)=(-1)^{k+1} .
$$


Proposition 4.1 is not difficult to prove directly. For a more general result, see [24, p. 122].

We shall consider the action of group $G$ of isometries of the $n$-cube $C_{n}$, a finite subgroup of the orthogonal group $O(n)$. Evidently, $G$ acts transitively on the set of $i$ dimensional faces of $C_{n}$ for each $i=0,1, \ldots, n$. It is not difficult to show that $|G|=2^{n} n$ !, while the orbit $U_{i}$ of an $i$-dimensional face has size

$$
\left|U_{i}\right|=\left(\begin{array}{c}
n \\
i
\end{array}\right) 2^{n-i}
$$

Let $J\left(C^{n}\right)$ denote the lattice of order ideals in $C^{n}$. The Basis Theorem 2.3 implies that if $\varphi$ is an invariant valuation on $J\left(C^{n}\right)$, then

$$
\varphi=\sum_{i=0}^{n} c_{i} \varphi_{i}
$$

where $\varphi_{i}$ is the invariant valuation given by

$$
\varphi_{i}(A)=\left|A \cap U_{i}\right|
$$

for all $A \in J\left(C^{n}\right)$.

Kinematic formulas are now derived in the usual way. Suppose $\varphi$ is an invariant valuation on $J\left(C^{n}\right)$. For all $A, B \in J\left(C^{n}\right)$, we have the general kinematic formula

$$
\frac{1}{2^{n} n !} \sum_{g} \varphi(A \cap g B)=\sum_{i=0}^{n}\left(\begin{array}{c}
n \\
i
\end{array}\right)^{-1} 2^{i-n} c_{i} \varphi_{i}(A) \varphi_{i}(B),
$$

where $c_{i}=\varphi\left(\left\{C_{i}\right\}\right)$.

Suppose $\varphi$ is chosen to be the Euler characteristic $\chi$ for the lattice $J\left(C^{n}\right)$. For $0 \leq i \leq n$, the value $c_{i}$ is then given by

$$
c_{i}=\varphi\left(\left\{C_{i}\right\}\right)=-\mu\left(\emptyset, C_{i}\right)=(-1)^{i} .
$$

This follows from Proposition 4.1.

Combining this with (4.13), we obtain the principal kinematic formula

$$
\frac{1}{2^{n} n !} \sum_{g} \chi(A \cap g B)=\sum_{i=0}^{n}\left(\begin{array}{c}
n \\
i
\end{array}\right)^{-1} 2^{i-n}(-1)^{i} \varphi_{i}(A) \varphi_{i}(B) .
$$

If $A=\bar{C}_{k}$ and $B=\bar{C}_{l}$ are principal ideals, then the formula (4.14) becomes

$$
\frac{1}{2^{n} n !} \sum_{g} \chi(A \cap g B)=\sum_{i=0}^{n}(-1)^{i}\left(\begin{array}{l}
n \\
i
\end{array}\right)^{-1}\left(\begin{array}{l}
k \\
i
\end{array}\right)\left(\begin{array}{l}
l \\
i
\end{array}\right) 2^{k+l-i-n},
$$

giving the probability that a random $l$-dimensional face of $C_{n}$ meets a given $k$-dimensional face. It follows that there are

$$
\left(\begin{array}{l}
n \\
l
\end{array}\right) 2^{n-l} \sum_{i=0}^{n}(-1)^{i}\left(\begin{array}{l}
n \\
i
\end{array}\right)^{-1}\left(\begin{array}{l}
k \\
i
\end{array}\right)\left(\begin{array}{l}
l \\
i
\end{array}\right) 2^{k+l-i-n}=\sum_{i=0}^{n}(-1)^{i}\left(\begin{array}{l}
n \\
i
\end{array}\right)^{-1}\left(\begin{array}{l}
n \\
l
\end{array}\right)\left(\begin{array}{l}
k \\
i
\end{array}\right)\left(\begin{array}{l}
l \\
i
\end{array}\right) 2^{k-i}
$$


$l$-faces of $C_{n}$ that meet $C_{k}$.

Instead, if we apply the formula (4.13) to the case $\varphi=\varphi_{i}$ for a particular fixed $i \in\{0,1, \ldots, n\}$, we have

$$
\frac{1}{2^{n} n !} \sum_{g} \varphi_{i}(A \cap g B)=\left(\begin{array}{c}
n \\
i
\end{array}\right)^{-1} 2^{i-n} \varphi_{i}(A) \varphi_{i}(B),
$$

for all $A, B \in J\left(C^{n}\right)$. If $A=\bar{C}_{k}$ and $B=\bar{C}_{l}$ are principal ideals, then the formula (4.17) becomes

$$
\frac{1}{2^{n} n !} \sum_{g} \varphi_{i}\left(\bar{C}_{k} \cap g \bar{C}_{l}\right)=2^{k+l-n-i}\left(\begin{array}{l}
n \\
i
\end{array}\right)^{-1}\left(\begin{array}{l}
k \\
i
\end{array}\right)\left(\begin{array}{l}
l \\
i
\end{array}\right),
$$

giving the expected number of $i$-faces of the intersection of a given $k$-face of $C_{n}$ with a random $l$-face of $C_{n}$.

Using the definition of expectation and standard probabilistic arguments, one can compute the left-hand sides of (4.15) and (4.18) more directly. In this case, powers of 2 appearing on both sides of these expressions cancel out, resulting once again in the same identities (4.4) and (4.5) obtained from the example of finite Boolean algebras.

\section{Variations and Generalizations}

There remain many other variations and applications of the general kinematic formula, Theorem 3.1. For example, one can replace the random intersection of two order ideals with a random union. For any invariant valuation $\varphi$ on $J(P)$, the valuation identity (1.1) implies that

$$
\frac{1}{|G|} \sum_{g \in G} \varphi(A \cup g B)+\frac{1}{|G|} \sum_{g \in G} \varphi(A \cap g B)=\varphi(A)+\varphi(B) .
$$

A kinematic formula for the expected value of a random union, $\varphi(A \cup g B)$, is then derived from a combination of Theorem 3.1 with the identity (5.1).

One can also consider multiple intersections. The proof of Theorem 3.1 easily generalizes to the case of multiple intersections to yield the kinematic formula:

$$
\frac{1}{|G|^{k}} \sum_{g_{1}, \ldots, g_{k} \in G} \varphi\left(A \cap g_{1} B_{1} \cap \cdots \cap g_{k} B_{k}\right)=\sum_{U \in \mathcal{U}} \frac{1}{|U|^{k}} c_{U} \varphi_{\mathrm{U}}(A) \varphi_{\mathrm{U}}\left(B_{1}\right) \cdots \varphi_{\mathrm{U}}\left(B_{k}\right),
$$

for all order ideals $A, B_{1}, \ldots, B_{k} \in J(P)$. Similarly, one can derive kinematic formulas for multiple unions.

\section{References}

1. W. Blaschke, Vorlesungen über Integralgeometrie, 3rd Ed., VEB Deutsch. Verlag d. Wiss., Berlin, 1955. (Also: First Edition Part I, 1935; Part II, 1937).

2. W.Y.C. Chen and G.-C. Rota, $q$-Analogs of the inclusion-exclusion principle, Discrete Math. 104 (1992) 7-22. 
3. S.S. Chern, On the kinematic formula in the euclidean space of $n$ dimensions, Amer. J. Math. 74 (1952) 227-236.

4. J.H.G. Fu, Kinematic formulas in integral geometry, Indiana Univ. Math. J. 39 (1990) 1115 1154.

5. S. Glasauer, Translative and kinematic integral formulae concerning the convex hull operation, Math. Z. (to appear).

6. J. Goldman and G.-C. Rota, On the foundations of combinatorial theory. IV. Finite vector spaces and Eulerian generating functions, Studies in Appl. Math. 49 (1970) 239-258.

7. H. Hadwiger, Vorlesungen über Inhalt, Oberfläche, und Isoperimetrie, Springer Verlag, Berlin, 1957.

8. R. Howard, The kinematic formula in Riemannian homogeneous spaces, Memoirs of the A.M.S. 509 (1993).

9. D. Klain, A short proof of Hadwiger's characterization theorem, Mathematika 42 (1995) 329-339.

10. D. Klain, Kinematic formulas for finite vector spaces, Discrete Math. (to appear).

11. D. Klain and G.-C. Rota, Introduction to Geometric Probability, Cambridge University Press, New York, 1997.

12. J. Kung, Ed., Gian-Carlo Rota on Combinatorics: Introductory Papers and Commentaries, Birkhäuser Verlag, Boston, 1995.

13. P. McMullen, Non-linear angle-sum relations for polyhedral cones and polytopes, Math. Proc. Camb. Phil. Soc. 78 (1975) 247-261.

14. P. McMullen, Valuations and dissections, In: Handbook of Convex Geometry, Peter M. Gruber and Jörg M. Wills, Eds., North-Holland, Amsterdam, 1993, pp. 933-988.

15. P. McMullen and R. Schneider, Valuations on convex bodies, In: Convexity and its Applications, Peter M. Gruber and Jörg M. Wills, Eds., Birkhäuser Verlag, Boston, 1983, pp. 170-247.

16. N. Metropolis and G.-C. Rota, Combinatorial structure of the faces of the $n$-cube, SIAM J. Appl. Math. 35 (1978) 689-694.

17. N. Metropolis, G.-C. Rota, V. Strehl, and N. White, Partitions into chains of a class of partially ordered sets, Proc. Amer. Math. Soc. 71 (1978) 193-196.

18. G.-C. Rota, On the foundations of combinatorial theory, I: Theory of Möbius functions, Z. Wahrscheinlichkeitstheorie 2368 (1964) 340-368.

19. G.-C. Rota, On the combinatorics of the Euler characteristic, Studies in Pure Math., (Papers Presented to Richard Rado) Academic Press, London, 1971, pp. 221-233.

20. G.-C. Rota, Introduction to Geometric Probability, Lezioni Lincee held at the Scuola Normale Superiore Pisa, December 2-22, 1986.

21. L.A. Santaló, Integral Geometry and Geometric Probability, Reading, Addison-Wesley, MA, 1976.

22. R. Schneider, Convex Bodies: The Brunn-Minkowski Theory, Cambridge University Press, New York, 1993.

23. R. Schneider and J.A. Wieacker, Integral geometry, In: Handbook of Convex Geometry, Peter M. Gruber and Jörg M. Wills, Eds., North-Holland, Amsterdam, 1993, pp. 1349-1390.

24. R. Stanley, Enumerative Combinatorics, Wadsworth \& Brooks/Cole Advanced Books and Software, Monterey, Calif., 1986.

25. J.H. van Lint and R.M. Wilson, A Course in Combinatorics, Cambridge University Press, New York, 1992.

26. W. Weil, Kinematic integral formulas for convex bodies, In: Contributions to Geometry, Proc. Geometry Symp., Siegen, 1978, J. Tölke and Jörg M. Wills, Eds., Birkhäuser Verlag, Boston, 1978, pp. 60-76.

27. G. Zhang, Dual kinematic formulas, Trans. Amer. Math. Soc. (to appear). 